\title{
Mortality Rate and Outcome among Patients Admitted to General Intensive Care Unit during "Morning-Hour” Compared with "Off-Hour"
}

\author{
Shahram Ala ${ }^{1,2}$, Nasrin Pakravan ${ }^{1,2 *}$, Motahhare Ahmadi ${ }^{2}$ \\ ${ }^{1}$ Imam Khomeini Teaching Hospital, Mazandaran University of Medical Science, Sari, Iran; ${ }^{2}$ Faculty of Pharmacy, Mazandaran \\ University of Medical Science, Sari, Iran. \\ Email: "npakravan@mazums.ac.ir, dr_p_n@yahoo.com
}

Received January $17^{\text {th }}$, 2012; revised February 25 ${ }^{\text {th }}$, 2012; accepted March $29^{\text {th }}$, 2012

\begin{abstract}
Aim: This study aim to evaluate the effect of time of admission on mortality of patients admitted to the ICU. Method: This retrospective study included 391 of patients admitted to the ICU of an academic hospital during one year. Patients were categorized according to time of admission: working-hours and off-hours. Mortality was compared in the groups and associated factors of mortality were examined. Results: Two third of patients were admitted during off-hours. There was no significant difference in the GCS, age and length of stay among patients admitted during working-hours and off-hours. There was no difference in mortality among patients admitted during working-hours and off-hours Mortality was significantly higher in older and more severe cases, regardless of time of admission. Conclusion: Time of ICU admission does not have significant effect on patient's outcome.
\end{abstract}

Keywords: Critical Care; Intensive Care Unit; ICU; Mortality

\section{Introduction}

Patients admitted to the internal care unit (ICU) often have multi organ failure and different abnormalities that require special care. The availability of appropriate diagnostic test and treatment is more imperative in the first few hours after admission, when patients are more likely to be critically ill, unstable and require vigorous care [1, 2]. Several cohort studies have demonstrated that ICU staffing may affect patients' outcome. High-intensity physician staffing (mandatory intensivist consultation or closed ICU) reduces ICU mortality and lengths of stay when compared with low-intensity physician staffing (no intensivist or elective intensivist consultation) [2]. Staffing levels in ICU tend to be lower during evening-night, weekend and holiday shifts (referred as "off hours") compared to the morning shift during weekday (referred as "day shift" or "morning hours" or "working hours"). This might be most important for the first few hours after admission. Within this time the initial diagnosis are made and the treatment plan are formulated $[3,4]$.

Because of lower staffing levels in the ICU during "off-hours" most of the mortalities may occur in this period [2,5]. Recent studies have shown the relationship between the time of admission and patient's outcome

\footnotetext{
*Corresponding author.
}

with contradictory results [3,6-13]. While some studies showed a poor outcome for patients admitting during offhours $[7,14]$. other reported a surprising better outcome $[1,2]$. This discrepancy might be in part due to differences in case mixes, number of medical personnel, and the available technology among different studies. Additionally, not all studies have taken into consideration the presence of important confounding factors, including: patient's origin, co-morbidities, and the intensity of care during off-hours [3]. If patient presenting during off-hour have a poor outcome, this would have serious implications for health-care administrator and insurance provider. The goal of the current study was to investigate the effect of time of ICU admission on ICU mortality among patients admitted to the general ICU of a Hospital in Northern Iran. It was hypothesized that mortality might be higher in off-hour when lower personnel are present.

\section{Method}

In an attempt to compare the mortality rate and time of admission to the ICU: off-hour, working-hour, and the possible effect of fewer ICU medical personnel and other associated factors on mortality, a retrospective study was conducted based on prospectively recorded data from patients admitted to the general ICU of a Teaching Hos- 
pital in Iran during one-year from $21^{\text {st }}$ of March 2008 to $20^{\text {th }}$ of March 2009. General ICU in the mentioned Teaching hospital includes nine beds. Adult ill patients over 16 year-old with different medical problems that required intensive care were admitted to this unit. In just few cases patient under 16 year-old were also admitted in this unit. Patients with surgical problems and post-operative were admitted to the surgical ICU. However, patients with internal neurosurgical and orthopedic problems were also admitted to the general ICU.

In Iran, week days are defined by law as Saturday till Thursday, and Friday is considered weekend holiday. There are also two weeks new-year holiday and other public holidays. During one year round from March $21^{\text {st }}$ 2008 to March $20^{\text {th }} 2009$, 78 days were holiday in total, including weekend, new year holiday and public holidays. According to the studied Teaching hospital's regulation morning shift is started from seven am to two pm; evening shift from one pm to eight pm and night shift from seven pm to eight am. One hour from seven am-eight am, one pm-two pm and seven pm-eight pm is considered as handover time when the ICU personnel from previous and next shift are both present. In the morning shift of week days, ICU personnel consisted of one board certified anesthesiologist physician, one medical resident, six critical care nurses and two trained ICU technicians. In the morning shift of the weekend and public holidays five critical care nurses are present instead of six. The number of ICU staff in the evening and night shifts of week day, weekend or public holiday include one board certified anesthesiologist physician, one medical resident, two trained ICU technicians but only four critical care nurses. During each shift the senior intensivist, an anesthesiologist physician leading the team, was fully responsible for the ICU care. Recorded data include age, gender, date and time of admission, GCS (Glasgow scale score), length of ICU stay, outcome (discharge from ICU or died in the ICU) and primary reason for admission. The primary reasons for admission to the ICU were classified as neurosurgery, toxicology and poisoning, respiratory, renal, hematology-oncology, orthopedics, gastrointestinal, obstetric and others (cardiovascular, infection, endocrine, urology, internal, neurology and surgery).

Patients were categorized according to the date and time of admission into two groups: working-hour and offhours admission). Working-hours group included admissions during morning shifts of the working days between eight am and two pm; and off-hour group included admissions in the evening and night shift (between two pm and eight am) in the week days or admissions at any time during the weekend and holiday. Because the presence of both previous and next shift personnel during handover time thus outcome measure (death and discharge) of pa- tients admitted during handover time were excluded from the comparison analyses, however, there was no death recorded during handover time.

Because of the retrospective nature of the study some of the above information for few patients was missing. Time of admission in 33 subjects was missing. To assign an admission group for the missing 16 subjects randomly assigned in working-hours admission category and 17 subjects were classified in the off-hours admission group. Information for gender in three subjects was missing, of those two subjects were randomly considered as male and one as female.

\section{Data Analysis}

Results are expressed as mean \pm SD (standard deviation) and percentage. Unpaired Student t-test and chi-square tests were used for comparisons of continuous and nominal data, respectively. Prognostic variables associated with mortality in the univariable analysis with $\mathrm{p}<0.1$ were entered into a multivariable binary logistic - regression model using backward stepwise variable elimination ( $\mathrm{p}<$ $0.05)$ to estimate the odds ratios (OR) for in-ICU death. Statistical analysis was performed using SPSS 13 (SPSS, Chicago, IL) software. The significance level was defined as $\mathrm{p}<0.05$.

\section{Results}

During one year period 391 patients were admitted to the intensive care unit. Of those $37.6 \%$ were admitted during working-hour and $62.4 \%$ in the off-hour. 241 (61.6\%) subjects were men and 150 women (38.4. Mean age $( \pm$ SD) for men was $46.8 \pm 23.9$ and for women $50.3 \pm$ 22.4. Patients aged 20 - 29 year-old (19.4\%) and $70-79$ year-old $(14.6 \%)$ were the most common age group admitted to the ICU (Table 1). At the time of admission to the ICU the majority of patients (63\%) had fairly good

Table 1. Groups according to the age of patients admitted to the ICU.

\begin{tabular}{ccc}
\hline Age Group & Frequency & Percent \\
\hline$<20$ & 44 & 11.3 \\
$20-29$ & 79 & 20.2 \\
$30-39$ & 38 & 9.7 \\
$40-49$ & 43 & 11.0 \\
$50-59$ & 42 & 10.7 \\
$60-69$ & 43 & 11.0 \\
$70-79$ & 57 & 14.6 \\
$80-89$ & 40 & 10.2 \\
$90-99$ & 4 & 1.0 \\
$>=100$ & 1 & 0.3 \\
\hline
\end{tabular}

Total number: 391. 
condition with GCS $>12.4 .9 \%$ of patients had poor condition with GCS $<=3$. GCS was $4-6$ in $14 \%, 7-9$ in $11.9 \%$, and $10-12$ in $6.2 \%$ of the admitted patients (Table 2). The primary reason for the ICU admission in about one third of patients was due to neurosurgical problems, followed by poisoning (16.2\%) and respiratory failure (12.9\%) (Table 3).

Of 391 subjects 280 patients (71.6\%) were discharged from ICU and 111 subjects (28.4\%), including 60 men and 51 women died. GCS of patients who died was significantly lower and stayed longer in the ICU than those who survived ( $\mathrm{p}<0.0001)$. GCS in the death group was $8.7 \pm 0.4$ (mean \pm SEM: standard error of the mean) versus $12.4 \pm 0.2$ in the discharged group. Length of stay in

Table 2. Frequency of patients according to GCS.

\begin{tabular}{ccc}
\hline GCS & Frequency & Valid Percent \\
\hline 3 & 19 & 4.9 \\
4 & 15 & 3.8 \\
5 & 19 & 4.9 \\
6 & 20 & 5.1 \\
7 & 17 & 4.3 \\
8 & 22 & 5.6 \\
9 & 7 & 1.8 \\
10 & 14 & 3.6 \\
11 & 7 & 1.8 \\
12 & 3 & 0.8 \\
13 & 102 & 26.1 \\
14 & 12 & 3.1 \\
15 & 128 & 32.7 \\
\hline
\end{tabular}

Total $\mathrm{n}=383$, missing data: 5 .

Table 3. Frequency of ICU admission and mortality in the patient groups according to the primary reason of ICU admission.

\begin{tabular}{|c|c|c|c|}
\hline $\begin{array}{l}\text { Primary Reason } \\
\text { For Admission }\end{array}$ & Frequency & Percent & Valid Percent \\
\hline Neurosurgery & 118 & 30.2 & 32.3 \\
\hline Toxicology & 59 & 15.1 & 16.2 \\
\hline Respiratory & 47 & 12.0 & 12.9 \\
\hline Hematology & 31 & 7.9 & 8.5 \\
\hline Renal & 30 & 7.7 & 8.2 \\
\hline Orthopedic & 16 & 4.1 & 4.4 \\
\hline Obstetric & 13 & 3.3 & 3.6 \\
\hline Gastrointestinal & 12 & 3.1 & 3.3 \\
\hline Others ${ }^{*}$ & 39 & 10 & 10.7 \\
\hline
\end{tabular}

Total: $\mathrm{n}=391$; Missing data: $\mathrm{n}=26$; "Others include: cardiovascular, infection, endocrine, urology, internal, neurology and surgery the ICU was $13.8 \pm 1.8$ day (mean \pm SEM) in the death group and $7.3 \pm 0.6$ day in the survived group. Mortality in women was slightly higher (34\% in women vs. $4.9 \%$ in men). The gender difference in mortality was in a borderline significant difference $(p=0.05)$. GCS and length of ICU stay for men and women were $11.2 \pm 0.3$ vs. $11.5 \pm 0.3(\mathrm{p}=0.6)$, and $9.4 \pm 0.9$ vs. $8.7 \pm 1.1$ day (p $=0.5$ ), respectively. There was no significant difference in the GCS and length of ICU stay between men and women.

147 patients were admitted during the working-hours and 244 in the off-hours (Table 4). There was no significant difference in the GCS, age and length of ICU stay among patients admitted during working-hours and offhours. Mean ( \pm sem) for the age, length of stay, and GCS was $46.5 \pm 1.9$ vs. $49.1 \pm 1.5$ year ( $p=0.3), 7.9 \pm 0.8$ vs. $9.9 \pm 1.0$ day ( $\mathrm{p}=0.1$ ), and $11.5 \pm 0.32$ vs. $11.2 \pm 0.26$ (p $=0.5$ ) in the working-hour and off-hour group, respecttively. There was no significant difference in mortality among patients admitted during working-hours and offhours ( $\mathrm{p}=0.4)$. The mortality was $25.9 \%(38 / 147)$ in the working-hour admission group and $29.9 \%$ (73/244) in the off-hours admitted group (Table 4). There was no difference in mortality among patients admitted in the morning, evening and night, and weekend and holiday ( $\mathrm{p}$ $=0.046)$. Mortality was $25.9 \%$ (38/147), 31.6\% (54/171) and $26 \%(19 / 73)$ in patients admitted during workinghours, evening and night, and weekend and holiday, respectively.

In an attempt to explore the associated factors related to the mortality backward stepwise regression was performed. Factors including: gender, age, GCS at admission to the ICU, length of stay in the ICU and time of admission (working-hours or off-hours) were entered into the model; of those GCS at admission and age were found to significantly affect the outcome ( $<<0.0001)$. Mortality was higher in patients who were older and had lower GCS at admission. The effect of gender on outcome was borderline and tend to be higher among women $(\mathrm{p}=0.07)($ Table 5).

\section{Discussion}

The organizational and staffing structure of an ICU influences the outcome of critically ill patients. A change in the ICU staffing structure frequently occurs at evening, night time and on weekends and holidays (off-hours). Thus, patients who are admitted to an ICU during offhours may be at higher risk of mortality. This hypothesis has been examined previously by several cohort studies. The results, however, have been contradictory. Whereas some studies reported an increased risk of death among patients admitted during off-hours [7,14], others reported a surprising protective effect $[9,10]$. 
Table 4. Demographic characteristic of the study population according to the time of admission.

\begin{tabular}{|c|c|c|c|}
\hline Parameter & $\begin{array}{l}\text { Working-hours admission } \\
\text { n (\%): } 147(37.6 \%)\end{array}$ & $\begin{array}{l}\text { Off-hours admission } \\
\text { n (\%): } 244(62.4 \%)\end{array}$ & p Value \\
\hline Gender (M/F) & $88 / 59$ & $153 / 91$ & 0.5 \\
\hline Age y & $46.5 \pm 1.9$ & $49.1 \pm 1.5$ & 0.5 \\
\hline GCS $^{*}$ & $11.5 \pm 0.32$ & $11.2 \pm 0.26$ & 0.5 \\
\hline ICU Stay (day) & $7.9 \pm 0.8$ & $9.9 \pm 1.0$ & 0.1 \\
\hline In-ICU Mortality n (\%) & $38(25.9 \%)$ & $73(29.9 \%)$ & 0.4 \\
\hline \multicolumn{4}{|c|}{ Reasons for admission (valid percent/number in each group) ${ }^{* *}$} \\
\hline Neurosurgery & 42 & 76 & \\
\hline Respiratory & 16 & 31 & \\
\hline Toxicology & 26 & 33 & \\
\hline Hematology & 8 & 23 & \\
\hline Renal & 11 & 19 & \\
\hline Orthopedic & 4 & 12 & \\
\hline Obstetric & 6 & 7 & \\
\hline Gastro-intestinal & 8 & 4 & \\
\hline Others & 17 & 22 & \\
\hline \multicolumn{4}{|c|}{ Mortality according to the reason for admission ( ${ }^{* *}$ of death / $\mathrm{n}$ of admission), $\%$} \\
\hline Neurosurgery & 6/42 (14.3\%) & $17 / 76(22.4 \%)$ & \\
\hline Respiratory & 4/16 (25.0\%) & 11/31 (35.5\%) & \\
\hline Toxicology & 3/26 (11.5\%) & 6/33 (18.2\%) & \\
\hline Hematology & $5 / 8$ (62.5\%) & $12 / 23(52.2 \%)$ & \\
\hline Renal & 6/11 (54.5\%) & 8/19 (42.1\%) & \\
\hline Orthopedic & $0 / 4(0 \%)$ & 0/12 (0\%) & \\
\hline Obstetric & $1 / 6(16.6 \%)$ & 0/7 (0\%) & \\
\hline Gastro-intestinal & $1 / 8(14.5 \%)$ & $3 / 4(75.0 \%)$ & \\
\hline Others $^{* *+}$ & 7/17 (41.2\%) & $7 / 22$ (31.2\%) & \\
\hline
\end{tabular}

Total: $391 ;{ }^{*}$ GCS at the time of ICU admission (mean \pm standard error of the mean); ${ }^{* *}$ n: number; ${ }^{* *+}$ Others include: cardiovascular, infection, endocrine, urology, internal, neurology and surgery; Values are mean \pm standard error of the mean.

Table 5. Associated factors of mortality in the ICU.

\begin{tabular}{ccccccc}
\hline Variables entered & B & S.E. & Sig. & Exp(B) & \multicolumn{2}{c}{$95.0 \%$ C.I. for EXP(B) } \\
\hline GCS & -0.11 & 0.027 & 0.67 & 0.989 & 0.937 & Lower \\
Age & 0.022 & 0.006 & 0.000 & 1.022 & 1.010 & 1.034 \\
ICU Stay & 0.024 & 0.010 & 0.012 & 1.024 & 1.005 & 1.044 \\
Gender (male) & -0.486 & 0.270 & 0.072 & 0.615 & 0.362 & 1.045 \\
Constant & 0.500 & 0.493 & 0.311 & 1.649 & & \\
\hline
\end{tabular}

Binary Logistic Regression (Backward Stepwise) was performed. Factors entered in eth model: gender, age, GCS at admission to the ICU, length of stay in the ICU, time of ICU admission (working-hour or off-hour). 
In the current study we evaluated the effect of time of admission on patients admitted to a general ICU in Northern Iran. The study showed that almost two third of the patients were admitted during off-hours (evening and night shift, weekend and public holidays), when fewer medical staff were present. No significant differences in age, GCS at admission, length of stay and ICU mortality of patient admitted during working-hours and off-hours were found, reflecting that patients admitted during both shifts had almost similar severity of illness.

The results of the current study are consistent with the findings of the previous studies in which no significant effect of time of admission on outcome were reported $[10,12,15]$. Moral and colleagues investigated the relationship between hospital mortality, length of stay and night time ICU admission, and showed no association [9].

It has been also reported that adults with serious medical conditions are more likely to die in the hospital if they are admitted on a weekend than if they are admitted on a weekday $[7,11,16-18]$. Cram and colleagues showed that the weekend effect on outcome was larger in major teaching hospitals and is cause for concern [17]. Barnett et al. also reported the same result, but they argue that, the similar risk for death in patients admitted on Friday and Monday suggests that "weekend effects" may be associated with unmeasured severity of illness and/or selection bias than to differences in quality of care [7]. In the current study we also examined the effect of week day and weekend or holiday admission on outcome, and no significant association was found. The same result was also reported by others $[6,8,12]$.

The presence of an intensivist has been reported to be an important factor on ICU mortality. A study in the UK evaluating off-hour consultant coverage found that the mortality rate was lower for patients admitted to the ICU after the introduction of 24-hour intensivist coverage [19]. Goh and colleagues et al. confirmed those results for the pediatric ICU; 24-hour availability of intensivist was associated with improved outcome and use of resources [20]. Another study on a cohort of patients admitted to the pediatric ICU showed that lack of in-house intensivist presence is not associated with mortality; on the contrary, off-hours admission decreased risk-adjusted mortality and length of stay on the population studied [10]. In a large sample of ICU patients Levy and colleagues compared the hospital outcome between subjects cared for entirely by critical care physicians and patients cared for entirely by non-critical care physicians. Surprisingly, they reported that the odds of hospital mortality were higher for patients who were treated by ICU physician than those who were not [21]. Another study showed the same reverse result and the author explained that the lower percentage of patient transferred during off-hour, who might have ill condition, might be an explanation for the lower mortality among patient admitted during off-hour $[3,22]$. In the current study there was an on-site board certified anesthesiologist in each shift, and therefore we were not able to compare the ICU mortality when the intensivist is present on site or on call. We, however, assume the presence of 24 hour anesthesiologist play an important rule on having a better outcome either during working hour or during off-hours when fewer nursing staffs are present.

Other studies reported that for ICUs, night-shift admission did not affect mortality than day time admission $[9,11,12]$ and surprisingly might even be associated with lower death rate [9]. There are several explanations for these observations. Firstly, diurnal organization of hospitals (rounds, orders, examinations, secretarial, administrative, and teaching responsibilities; interactions with patient's families) has a negative effect on the quality of patient's care [3]. Secondly, nursing workloads might be heavier relative to the nurse/patient staffing ratio during the daytime resulting in diverted attention and energies and contributing unintentionally to higher mortality [2325]. Finally, performing more invasive procedure and surgeries, and diagnostic interventions during the day time, each of which is accompanied by its own morbidity or mortality [26]. In our ICU like other ICUs more aggressive interventional diagnostic and therapeutic procedures are performed during the day, however, no significant difference in mortality between day and night admissions was observed.

There has been a consensus about the "golden hour" in ill patients admitting in the ICU. Timely intervention improves the outcomes of life-threatening illness; however, in the teaching hospitals because of the morning rounds at fixed times of the day patient's care is sometimes interrupted by educational sessions and rounds, resulting in delayed resuscitation of critically ill patients admitted to the ICU during rounds and providing a potential explanation for the higher mortality of patients admitted during this time period. Afessa et al. examined the association between ICU admission during morning rounds and mortality [27]. Their study results showed that patients admitted to the ICU during morning rounds had higher severity of illness and mortality rates. No such an effect was found in the current study.

Further analysis in the current data showed that regardless of time of admission factors such as age, and severity of illness measured by GCS at admission were found to affect the outcome. Older patient and those who had more critical condition at their admission had poor outcome. This study like other retrospective studies has inherent limitations that should be mentioned. The study 
was performed retrospectively by using prospectively collected data and as expected there was some missing data for time of admission, although we tried to handle the missing data by randomly assigning particular group category. The severity of illness is an important factor on outcome and the severity was compared by the GCS at admission. Although there was no significant difference in the GCS of patients admitted in off-hours and workinghours, GCS might not be a good marker to show the severity of illness or confounding variables that might have been affected the outcome. We did not have all information available to measure the APACHI or SAPS score, but, it is unlikely to affect the outcome as other studies in which severity of illness were adjusted using APACHI or SAPS scores showed the similar results $[3,9]$.

\section{Conclusion}

A change in the ICU staffing structure frequently occurs at nighttime and on weekends (off-hours).The staffing structure of an ICU influences the outcome of critically ill patients. Previous studies examining the effect of time of admission on outcome have shown contradictory results. In this study ICU off-hours admission was not associated with a poor outcome than working-hours shift, consistent with a constant level of care, regardless of the time of admission.

\section{REFERENCES}

[1] J. L. Vincent, "Need for Intensivists in Intensive-Care Units,” Lancet, Vol. 356, No. 9231, 2000, pp. 695-696.

[2] P. J. Pronovost, D. C. Angus, T. Dorman, K. A. Robinson, T. T. Dremsizov and T. L. Young, "Physician Staffing Patterns and Clinical Outcomes in Critically Ill Patients: A Systematic Review," Journal of American Medical Association, Vol. 288, No. 17, 2002, pp. 2151-2162. doi:10.1001/jama.288.17.2151

[3] C. E. Luyt, A. Combes, P. Aegerter, B. Guidet, J. L. Trouillet, C. Gibert, et al., "Mortality among Patients Admitted to Intensive Care Units During Weekday Day Shifts Compared with 'Off' Hours," Critical Care Medicine, Vol. 35, No. 1, 2007, pp. 3-11. doi:10.1097/01.CCM.0000249832.36518.11

[4] E. Rivers, B. Nguyen, S. Havstad, J. Ressler, A. Muzzin, B. Knoblich, et al., "Early Goal-Directed Therapy in the Treatment of Severe Sepsis and Septic Shock," New England Journal of Medicine, Vol. 345, No. 19, 2001, pp. 1368-1377. doi:10.1056/NEJMoa010307

[5] R. L. Kane, T. A. Shamliyan, C. Mueller, S. Duval and T. J. Wilt, "The Association of Registered Nurse Staffing Levels and Patient Outcomes: Systematic Review and Meta-Analysis,” Medical Care, Vol. 45, No. 12, 2007, pp. 1195-1204. doi:10.1097/MLR.0b013e3181468ca3

[6] Y. Arabi, A. Alshimemeri and S. Taher, "Weekend and Weeknight Admissions Have the Same Outcome of Week- day Admissions to an Intensive Care Unit with Onsite Intensivist Coverage,” Critical Care Medicine, Vol. 34, No. 3, 2006, pp. 605-611.

[7] M. J. Barnett, P. J. Kaboli, C. A. Sirio and G. E. Rosenthal, "Day of the Week of Intensive Care Admission and Patient Outcomes: A Multisite Regional Evaluation," Medical Care, Vol. 40, No. 6, 2002, pp. 530-539. doi:10.1097/00005650-200206000-00010

[8] S. A. Ensminger, I. J. Morales, S. G. Peters, M. T. Keegan, J. D. Finkielman, J. F. Lymp, et al., "The Hospital Mortality of Patients Admitted to the ICU on Weekends," Chest, Vol. 126, No. 4, 2004, pp. 1292-1298. doi:10.1378/chest.126.4.1292

[9] I. J. Morales, S. G. Peters and B. Afessa, "Hospital Mortality Rate and Length of Stay in Patients Admitted at Night to the Intensive Care Unit," Critical Care Medicine, Vol. 31, Vol. 3, 2003, pp. 858-863.

[10] A. Numa, G. Williams, J. Awad and B. Duffy, “AfterHours Admissions Are Not Associated with Increased Risk-Adjusted Mortality in Pediatric Intensive Care,” Intensive Care Medicine, Vol. 34, No. 1, 2008, pp. 148-151.

[11] A. Uusaro, A. Kari and E. Ruokonen, “The Effects of ICU Admission and Discharge Times on Mortality in Finland,” Intensive Care Medicine, Vol. 29, No. 12, 2003, pp. 2144-2148. doi:10.1007/s00134-003-2035-1

[12] H. Wunsch, J. Mapstone, T. Brady, R. Hanks and K. Rowan, "Hospital Mortality Associated with Day and Time of Admission to Intensive Care Units,” Intensive Care Medicine, Vol. 30, No. 5, 2004, pp. 895-901. doi:10.1007/s00134-004-2170-3

[13] R. Cavallazzi, P. E. Marik, A. Hirani, M. Pachinburavan, T. S. Vasu and B. E. Leiby, "Association between Time of Admission to the ICU and Mortality: A Systematic Review and Meta Analysis,” Chest, Vol. 138, No. 1, 2010, pp. 68-75.

[14] K. B. Laupland, R. Shahpori, A. W. Kirkpatrick and H. T. Stelfox, "Hospital Mortality among Adults Admitted to and Discharged from Intensive Care on Weekends and Evenings,” Journal of Critical Care, Vol. 23, No. 3, 2008, pp. 317-324. doi:10.1016/j.jcrc.2007.09.001

[15] Y. Numata, M. Schulzer, W. R. van der, J. Globerman, P. Semeniuk, E. Balka, et al., "Nurse Staffing Levels and Hospital Mortality in Critical Care Settings: Literature Review and Meta-Analysis," Journal of Advanced Nursing, Vol. 55, No. 4, 2006, pp. 435-448. doi:10.1111/j.1365-2648.2006.03941.x

[16] C. M. Bell and D. A. Redelmeier, "Mortality among Patients Admitted to Hospitals on Weekends as Compared with Weekdays," New England Journal of Medicine, Vol. 345, No. 9, 2001, pp. 663-668. doi:10.1056/NEJMsa003376

[17] P. Cram, S. L. Hillis, M. Barnett and G. E. Rosenthal, "Effects of Weekend Admission and Hospital Teaching Status on In-Hospital Mortality,” American Journal of Medicine, Vol. 117, No. 3, 2004, pp. 151-157. doi:10.1016/j.amjmed.2004.02.035

[18] J. B. Gould, C. Qin, A. R. Marks and G. Chavez, "Neonatal Mortality in Weekend vs Weekday Births,” Journal 
of American Medical Association, Vol. 289, No. 22, 2003, pp. 2958-2962. doi:10.1001/jama.289.22.2958

[19] M. C. Blunt and K. R. Burchett, "Out-of-Hours Consultant Cover and Case-Mix-Adjusted Mortality in Intensive Care,” Lancet, Vol. 356, No. 9231, 2000, pp. 735-736. doi:10.1016/S0140-6736(00)02634-9

[20] A. Y. Goh, L. C. Lum and M. E. bdel-Latif, "Impact of 24 Hour Critical Care Physician Staffing on Case-Mix Adjusted Mortality in Paediatric Intensive Care,” Lancet, Vol. 357, No. 9254, 2001, pp. 445-446. doi:10.1016/S0140-6736(00)04014-9

[21] M. M. Levy, J. Rapoport, S. Lemeshow, D. B. Chalfin, G. Phillips and M. Danis, “Association between Critical Care Physician Management and Patient Mortality in the Intensive Care Unit,” Annals of Internal Medicine, Vol. 148, No. 11, 2008, pp. 801-809.

[22] A. Combes, C. E. Luyt, J. L. Trouillet, J. Chastre and C. Gibert, "Adverse Effect on a Referral Intensive Care Unit's Performance of Accepting Patients Transferred from Another Intensive Care Unit," Critical Care Medicine, Vol. 33, No. 4, 2005, pp. 705-710. doi:10.1097/01.CCM.0000158518.32730.C5

[23] L. H. Aiken, S. P. Clarke and D. M. Sloane, "Hospital
Staffing, Organization, and Quality of Care: Cross-National Findings," Nursing Outlook Journal, Vol. 50, No. 5 2002, pp. 187-194. doi:10.1067/mno.2002.126696

[24] R. K. Amaravadi, J. B. Dimick, P. J. Pronovost and P. A. Lipsett, "ICU Nurse-to-Patient Ratio Is Associated with Complications and Resource Use after Esophagectomy," Intensive Care Medicine, Vol. 26, No. 12, 2006, pp. 18571862. doi:10.1007/s001340000720

[25] W. O. Tarnow-Mordi, C. Hau, A. Warden and A. J. Shearer, "Hospital Mortality in Relation to Staff Workload: A 4Year Study in an Adult Intensive-Care Unit,” Lancet, Vol. 356, No. 9225, 2000, pp. 185-189. doi:10.1016/S0140-6736(00)02478-8

[26] P. G. Metnitz, A. Reiter, B. Jordan and T. Lang, "More Interventions Do Not Necessarily Improve Outcome in Critically Ill Patients,” Intensive Care Medicine, Vol. 30, No. 8, 2004, pp. 1586-1593.

[27] B. Afessa, O. Gajic, I. J. Morales, M. T. Keegan, S. G. Peters and R. D. Hubmayr, "Association between ICU Admission during Morning Rounds and Mortality," Chest, Vol. 136, No. 6, 2009, pp. 1489-1495. doi:10.1378/chest.09-0529 